\title{
Factores predictivos de conversión en la sigmoidectomía laparoscópica para el tratamiento de la enfermedad diverticular*
}

\author{
Drs. ADRIÁN MURILlO Z. ${ }^{1}$, JAVIER ROBLES C. ${ }^{1}$, JOSÉ A. POSADA T. ${ }^{1}$, PABLO D. MURAKAMI M. ${ }^{1}$ \\ 1 Servicio de Cirugía General American British Cowdray Medical Center. I.A.P, México D.F., México.
}

\begin{abstract}
Predictive factors of conversion in laparoscopic sigmoidectomy in patients with diverticular disease

Laparoscopic surgery diminihes the morbidity of colorrectal surgery. These benefits are lost if the surgery is converted to an open procedure. We searched for predictive factors of conversion in patients with diverticular disease. A study of 79 patients who underwent laparoscopic sigmoid resection was performed, comparing those who underwent conversion and those who didn`t. Material and Methods: A retrospective cohort study was done in a third level hospital of the patients who required laparoscopic sigmoidectomy during the last 7 years. Analysis: The t Student, test and the exact Fisher test were used. We considered $p<0.05$ (95\% confidence interval) as statistically significant. Results: The pre operative variables of age, sex, BMI, ASA, previous abdominal surgery, complicated or uncomplicated diverticulitis, and type of surgery were considered. Post operative variables considered were operative time, bleeding, return of bowel function, and hospital stay. No factor was identified as predictive of conversion. There was a statistically significant difference between both groups when surgical time $(p=0.0030)$ and operative bleeding $(\mathrm{p}=0.0272)$ were compared. Conclusions: We failed to identify a single factor predictive of conversion to an open procedure. We think it is more probable that a confluence of different variables lead to this result. The patients in whom the conversion was performed had more bleeding and prolonged surgical times, which makes them more prone to post operative complications.
\end{abstract}

Key words: Diverticulitis, diverticular disease, laparoscopy.

\section{Resumen}

La laparoscopia disminuye la morbilidad de la cirugía colorrectal. Estos beneficios se pierden con la conversión. Buscamos factores predictivos de conversión en pacientes con enfermedad diverticular. Se realizó un estudio retrospectivo de 79 pacientes en quienes se realizó sigmoidectomía laparoscópica y se comparó los pacientes que requirieron conversión y los que no. Material y Métodos: Se hizo un estudio de cohorte retrospectivo en un hospital de tercer nivel de los pacientes a los que se realizó sigmoidectomía laparoscópica. Análisis: Se utilizó las pruebas t de Student y prueba exacta de Fisher. Se tomó como

*Recibido el 16 de Junio de 2009 y aceptado para publicación el 28 de Julio de 2009.

Correspondencia: Dr. Adrián Murillo Z.

Sur 136 No 116 Col. Las Américas

Delegación Alvaro Obregon. CP 01120 México D.F., México.

E-mail: dradrianmurillo@gmail.com 
estadísticamente significativo un valor de $\mathrm{p}<0,05$ (intervalo de confianza 95\%). Resultados: Se valoraron las variables pre operatorias de edad, sexo, IMC, clasificación ASA, cirugía abdominal previa, diverticulitis complicada o no complicada y tipo de cirugía. Variables post operatorias que se consideraron fueron tiempo quirúrgico, sangrado intra operatorio, retorno a función intestinal y estancia hospitalaria. No se identificó ningún factor predictivo de conversión. Si hubo diferencia estadísticamente significativa entre ambos grupos en cuanto al tiempo quirúrgico $(\mathrm{p}=0,0030)$ y al sangrado intra operatorio $(\mathrm{p}=0,0272)$. Conclusiones: No identificamos ningún factor predictivo de conversión en el tratamiento de la enfermedad diverticular por laparoscopia. Creemos que no hay un factor único que pueda ser utilizado para esto, más bien, es la confluencia de ciertas variables lo que conlleva a este resultado. Los pacientes en los que se realizó la conversión tienen mayor sangrado y tiempo quirúrgico, lo que pudiera hacer que sean más propicios a tener complicaciones post operatorias.

Palabras clave: Diverticulitis, enfermedad diverticular, laparoscopia.

\section{Introducción}

El tratamiento laparoscópico en la enfermedad colorrectal ha logrado disminuir la morbilidad asociada al evento quirúrgico ${ }^{1}$. La conversión a cirugía abierta hace que se pierdan estos beneficios. Específicamente en el tratamiento de la enfermedad diverticular la cirugía laparoscópica ha evolucionado y se encuentra en este momento en el punto en que el tratamiento de la diverticulitis complicada se considera factible. Esto a pesar del mayor grado de dificultad asociado al procedimiento bajo estas condiciones. Se han publicado diversas series donde se comparan los resultados del manejo laparoscópico de la diverticulitis no complicada con la complicada mostrando un índice de conversión a cirugía abierta similar entre ambos grupos ${ }^{2,3}$.

El propósito de este estudio fue tratar de identificar factores de riesgo pre operatorios para conversión a cirugía abierta en el tratamiento laparoscópico de la enfermedad diverticular y la diverticulitis. Así mismo se valoró los efectos de la conversión en los resultados a corto plazo. Se revisaron los expedientes de 79 pacientes operados durante un período de 7 años y se documentan los resultados.

\section{Material y Método}

Se realizó un estudio de cohorte retrospectivo en un hospital de tercer nivel privado en la Ciudad de México, donde se incluyó a todos los pacientes a quienes se realizó el diagnóstico de enfermedad diverticular o diverticulitis, que fueron sometidos a una sigmoidectomía laparoscópica, desde el 01 enero de 2002 hasta 01 enero de 2009. Se tomaron las variables de edad, género, índice de masa corporal, clasificación de la sociedad americana de anestesiología (ASA), cirugía abdominal previa, diver- ticulitis no complicada y complicada, tipo de cirugía (laparoscópica asistida y mano asistida), tiempo quirúrgico, sangrado intra operatorio, retorno a función intestinal (primer flato o evacuación) y estancia hospitalaria.

Se realizó una comparación entre aquellos pacientes que requirieron conversión a cirugía abierta y aquellos en los que su cirugía se completó de manera laparoscópica. Se evaluó factores pre operatorios que puedan estar relacionados con un mayor riesgo de conversión, y posteriormente se analizaron los efectos de la conversión en la evolución post operatoria del paciente.

\section{Análisis estadístico}

Se realizó un análisis comparativo entre ambos grupos. Se utilizó la prueba exacta de Fisher para comparar las variables de sexo, clasificación de ASA, cirugía previa, diverticulitis no complicada/ complicada y el tipo de cirugía. Se utilizó la prueba t de Student para comparar las variables de edad, IMC, tiempo quirúrgico, sangrado intra-operatorio, días hasta el retorno de la función intestinal y días de estancia hospitalaria post quirúrgica. Se tomó el valor de $\mathrm{p}<0,05$ (intervalo de confianza 95\%) como estadísticamente significativo.

\section{Resultados}

Se realizaron durante el período establecido 79 sigmoidectomías laparoscópicas. La edad promedio fue de 55,7 años de edad (rango 33-79). La proporción masculino: femenino fue de 2,16:1. El IMC promedio fue de $26,1 \mathrm{~kg} / \mathrm{m}^{2}$ (rango 19-38). Treinta y un pacientes fueron clasificados como ASA 1, 47 como ASA 2 y uno como ASA 3. Cuarenta y uno tenían antecedente de cirugía abdominal previa. Cincuenta y dos pacientes tenían diverticulitis no complicada y 27 tenían diverticulitis complicada. Se realizaron 38 cirugías laparoscópicas asistidas y 41 ci- 
FACTORES PREDICTIVOS DE CONVERSIÓN EN LA SIGMOIDECTOMÍA LAPAROSCÓPICA PARA EL ...

Tabla 1. Análisis comparativo de los factores de conversión de pacientes que no requirieron conversión a cirugía abierta y de aquellos que sí la requirieron

\begin{tabular}{|c|c|c|c|}
\hline & $\begin{array}{c}\text { Sin Conversión } \\
n=72(\%)\end{array}$ & $\begin{array}{c}\text { Conversión a cirugía abierta } \\
n=7(\%)\end{array}$ & $\mathbf{p}$ \\
\hline Edad (años) $)^{\mathrm{a}}$ & $55,4 \quad( \pm 11,3)$ & $58,9( \pm 17,3)$ & 0,4681 \\
\hline Masculino: Femenino ${ }^{b}$ & $48: 24$ & $6: 1$ & 0,4218 \\
\hline $\operatorname{IMC}\left(\mathrm{kg} / \mathrm{m}^{2}\right)^{\mathrm{a}}$ & $26,1( \pm 3,6)$ & $25,3( \pm 2,6)$ & 0,5445 \\
\hline Clasificación $\mathrm{ASA}^{\mathrm{b}}$ & & & 0,6983 \\
\hline 1 & $29(40 \%)$ & $2(29 \%)$ & \\
\hline$>1$ & $43(60 \%)$ & $5(71 \%)$ & \\
\hline Cirugía abdominal previa ${ }^{\mathrm{b}}$ & $37(51 \%)$ & $4(57 \%)$ & 1,0000 \\
\hline Diverticulitis $^{\mathrm{b}}$ & & & 0,6853 \\
\hline No complicada & $48(67 \%)$ & $4(57 \%)$ & \\
\hline Complicada & $24(33 \%)$ & $3(43 \%)$ & \\
\hline Tipo de cirugía ${ }^{\mathrm{a}}$ & & & 0,7053 \\
\hline Laparoscópica asistida & 34 & 4 & \\
\hline Mano asistida & 38 & 3 & \\
\hline
\end{tabular}

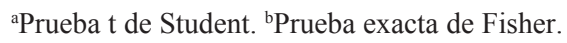

Tabla 2. Análisis comparativo de variables a corto plazo de pacientes que no requirieron conversión a cirugía abierta y de aquellos que si la requirieron

\begin{tabular}{|c|c|c|c|}
\hline & $\begin{array}{c}\text { Sin Conversión } \\
\mathrm{n}=72\end{array}$ & $\begin{array}{l}\text { Conversión a cirugía abierta } \\
\qquad \mathrm{n}=7\end{array}$ & $\mathbf{p}$ \\
\hline Tiempo quirúrgico $(\mathrm{min})^{\mathrm{a}}$ & $149 \quad( \pm 71,6)$ & $236 \quad( \pm 65,8)$ & 0,0030 \\
\hline Sangrado intra operatorio $(\mathrm{ml})^{\mathrm{a}}$ & $128 \quad( \pm 100,6)$ & $236 \quad( \pm 257,7)$ & 0,0272 \\
\hline Retorno a función intestinal (días) ${ }^{\mathrm{a}}$ & $1,9 \quad( \pm 0,9)$ & $2,4 \quad( \pm 0,8)$ & 0,1584 \\
\hline Estancia hospitalaria (días) ${ }^{\mathrm{a}}$ & $5,3 \quad( \pm 2,9)$ & $7,0 \quad( \pm 3,3)$ & 0,1417 \\
\hline
\end{tabular}

aPrueba t de Student.

rugías laparoscópicas mano asistidas.

El motivo de conversión fue en 4 pacientes adherencias que no se pudieron liberar, en uno hubo dehiscencia de la línea de anastomosis durante la cirugía, y en 2 no se logró visualizar de manera adecuada las estructuras.

En la Tabla 1 se muestran las características de los dos grupos y un análisis comparativo entre ellos. Ninguna de las variables analizadas en nuestro estudio tuvo impacto como factor pronóstico de conversión a un procedimiento abierto.

En la Tabla 2 se detallan las variables relacionadas a la recuperación post quirúrgica. Se muestra además la comparación entre ambos grupos. Tanto el tiempo quirúrgico como el sangrado intra operatorio fueron afectados de manera significativa por la conversión. Las variables de días a inicio de función intestinal y días de estancia hospitalaria tuvieron tendencia a ser más cortos en el grupo que no requirió conversión, sin embargo, no llegaron a ser estadísticamente significativas.

\section{Discusión}

La cirugía laparoscópica es una alternativa viable en el tratamiento de la enfermedad diverticular, tanto complicada como no complicada. En nuestra serie, el porcentaje de conversión fue $9 \%$ lo que está dentro del rango reportado por diversos auto- 
res $(2,1 \%-38 \%)^{4,5}$. La principal causa de conversión fue la presencia de adherencias intra abdominales. Esto concuerda con lo reportado en la literatura ${ }^{6}$. En nuestro análisis de las variables del paciente no encontramos ningún factor que pudiera funcionar como predictor de este tipo de evento.

Inclusive al comparar la cirugía mano asistida con la cirugía laparoscópica asistida no encontramos diferencia en el resultado. Este resultado no concuerda con lo reportado por diversos autores, donde se ha visto que una de las ventajas más importantes de la cirugía mano asistida es que se disminuye el índice de conversión ${ }^{7}$. Cabe hacer hincapié que en nuestra serie, 10 de los procedimientos documentados como mano asistidos comenzaron como procedimientos laparoscópicamente asistidos. En estos se optó por modificar el abordaje por dificultad en la exposición y/o manipulación de los tejidos. De no haber tenido la opción de la cirugía mano asistida, estos 10 procedimientos posiblemente hubieran terminado en conversión a cirugía abierta, lo que hubiera dado un índice de conversión mucho mayor para los procedimientos laparoscópicamente asistidos.

En la literatura hay trabajos que han tratado de identificar factores de riesgo para conversión, sin embargo, sus resultados no son homogéneos ${ }^{2,7,8}$. No descartamos la posibilidad que es el conjunto de varios factores los que pudiera favorecer la conversión.

Un factor que no fue analizado en este estudio y que está reportado como influyendo en el resultado, es la experiencia del cirujano. Esto principalmente se ha documentado en la cirugía laparoscópica asistida ya que la dificultad del procedimiento es mayor ${ }^{9,10}$.

Valoramos las variables a corto plazo de los pacientes en los que se logró completar la cirugía de manera laparoscópica y los que requirieron conversión. Evidentemente las personas que requirieron de conversión a cirugía abierta cursaron con un tiempo quirúrgico y un sangrado intra operatorio mayor. Ambos factores se han descrito como factores de riesgo que aumentan la morbilidad en el post operatorio $^{11,12}$. De ahí que creemos que estas cifras son importantes y podrían repercutir en la recuperación del paciente (complicaciones pulmonares, infección de herida quirúrgica).

Hubo una tendencia a que los pacientes en los que se logró completar el procedimiento de manera laparoscópica tuvieran un menor tiempo al inicio de la función intestinal y menor estancia hospitalaria, esto sin embargo, no fue estadísticamente significativo. Nosotros creemos que esto es en parte por el tamaño pequeño de nuestra muestra que requirió conversión a cirugía abierta. Otros autores documentan con resultados significativos que la conversión a un procedimiento abierto prolonga los días al retorno de la función intestinal y la estancia hospitalaria $^{8,13}$.

Creemos que la conversión a cirugía abierta refleja el buen juicio del cirujano. Sin embargo, se ha visto relacionada con un aumento importante de la morbilidad post operatoria cuando sucede ${ }^{14}$. Nosotros no logramos identificar ningún factor pre operatorio que pudiera funcionar como indicador de posible conversión. Lo que si observamos es que la conversión está relacionada a un sangrado intra operatorio y un tiempo quirúrgico mayor. Así mismo observamos una tendencia a que los pacientes en los que no se completó la cirugía de manera laparoscópica cursaran con una estancia hospitalaria más larga y un íleo más prolongado, lo que puede ser un reflejo de una evolución más lenta y/o complicaciones en el post operatorio.

\section{Referencias}

1. Schwenk W, Haase O, Neudecker JJ, Müller JM. Short term benefits for laparoscopic colorectal resection. Cochrane Database Syst Rev 20 (3) CD003145.

2. Hassan I, Cima RR, Larson DW, Dozois EJ, O'Byrne MM, Larson DR, et al. The Impact of Uncomplicated and Complicated Diverticulitis on Laparoscopic Surgery. Conversion Rates and Patient Outcomes. Surg Endosc 2007; 21: 1690-1694.

3. Reissfelder C, Buhr HJ, Ritz JP. Can laparoscopically assisted sigmoid resection provide uncomplicated management even in cases of complicated diverticulitis. Surg Endosc 2006; 20: 1055-1059.

4. Jones OM, Stevenson AR, Clark D, Stitz RW, Lumley JW. Laparoscopic Resection for Diverticular Disease. Follow-up of 500 Consecutive patients. Ann Surg 2008; 248: 1092-1097.

5. Gervaz P, Pikarsky A, Utech M, Secic M, Efron J, Belin B, et al. Converted laparoscopic colorectal surgery. Surg Endosc 2001; 15: 827-832.

6. Pandya S, Murria JJ, Coller JA, Rusia LC. Laparoscopic colectomy: indications for conversión to laparotomy. Arch Surg 1999; 134: 471-475.

7. Aalbers AG, Biere SS, van Berge Henegouwen MI, Bemelman WA. Hand assisted or laparoscopic assisted approach in colorrectal surgery: a systematic review and meta-analysis. Surg Endosc 2008; 22: 1769-1780.

8. Moine MC, Fabre JM, Vacher C, Navarro F, Picot $\mathrm{MC}$, Domergue J. Factors and consequences of conversion in laparoscopic sigmoidectomy for diverticular disease. Br J Surg 2003; 90: 232-236.

9. Targarona EM, García E, Rodríguez M, Cerdan G, Balague C, Garriaga J, et al. Hand assisted laparoscopic surgery. Arch Surg 2003; 138: 133-141. 
FACTORES PREDICTIVOS DE CONVERSIÓN EN LA SIGMOIDECTOMÍA LAPAROSCÓPICA PARA EL ...

10. Marusch F, Gastinger I, Schneider C, Scheidback H, Konradt J, Bruch HP, et al. Experience as a factor influencing the indications for laparoscopic colorectal surgery and the results. Surg Endosc 2001; 15: 116120.

11. Copeland GP, Jones D, Walters M. Possum: a scoring system for surgical audit. Br J Surg 1991; 78: 356360 .

12. Mitchell CK, Smoger SH, Pfeirer MP, Vogel RL, Pandit MK, Donnelly PJ, et al. Multivariate analysis of factors associated with postoperative pulmonary complications following general elective surgery. Arch Surg 1998; 133: 194-198.

13. Belizon A, Sardinha CT, Sher ME. Converted laparoscopic colectomy: what are the consequences? Surg Endosc 2006; 20: 947-951.

14. Marusch F, Gastinger I, Schneider C, Scheidback H, Konradt J, Bruch HP, et al. Importance of conversion for results obtained with laparoscopic colorectal surgery. Dis Colon Rectum 2001; 44: 207-214. 